\title{
Porous Silicon Based Sensor for Organic Vapors
}

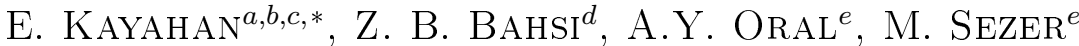 \\ ${ }^{a}$ Kocaeli University, Electro-Optics Systems Eng., Umuttepe, 41380, Kocaeli, Turkey \\ ${ }^{b}$ Kocaeli University, LATARUM Laboratory, 41275, Yenikoy, Kocaeli, Turkey \\ ${ }^{c}$ Kocaeli University, Hereke MYO, 41800, Hereke-Kocaeli, Turkey \\ ${ }^{d}$ Dept. of Environmental Engineering, Gebze Technical University, 41400, Kocaeli, Turkey \\ ${ }^{e}$ Dept. of Materials Science and Engineering, Gebze Technical University, 41400, Kocaeli, Turkey

\begin{abstract}
Porous silicon (PS) has been an attractive material for enhancing optical properties of silicon. Its large surface area for sensor applications and compatibility with silicon-based technologies has been a driving force for further technology development. In this study, ability of PS to sense at room temperature organic vapors such as acetone, trichloroethylene and hexane, which are harmful to human health, has been investigated. Electrical (DC) and photo-luminescence (PL) measurements in a controlled atmosphere (nitrogen gas and an organic vapor mix) were performed to test the sensor response towards the organic vapors. It was found that PS surface is very sensitive against these vapors. The experimental results also suggested that PS can be used as a new electro-optical material to sense harmful vapors.
\end{abstract}

DOI: 10.12693/APhysPolA.127.1400

PACS: 07.07.Df

\section{Introduction}

Acetone, trichloroethylene and n-hexane are organic compounds belonging to different organic groups. They are commonly used as industrial solvents, as compounds of glues and for cleaning purposes. Organic vapor sensors have been a very active research area, since organic vapors are among the most harmful gases [1]. Almost all organic vapors are potentially toxic to the liver and kidneys. Even brief exposure to these vapors can result in a variety of serious effects in both the peripheral nervous system and the central nervous system $[2,3]$. Organic vapors must be continuously monitored in our living environment. The traditional methods such as chromatography for detecting organic gases is costly, requires a relatively long time and needs professional laboratory facilities with specialized staff [4]. We also need new modern sensors cheap and capable of quickly sensing the organic vapors. Porous silicon-based vapor sensor could be the most attractive choice since it would be compact, sensitive and low cost. PS owing to its sponge-like structure has also attracted much attention due to a wide variety of possibilities for sensor applications [4]. Its large areato-volume ratio and surface bond configuration enhance sensing properties of PS against various chemical species.

Electrical methods, based on DC current measurements [5] and optical methods, based on photoluminescence modulation [6], reflectance [2], or interferometry [7] have also been proposed and very high sensitivities were demonstrated in sensing various organic vapors.

In this study, effects of the organic vapors (trichloroethylene, hexane and acetone) on photo- luminescence (PL) spectra and DC electrical properties of porous silicon were investigated. Possibility of an electrical and optical organic vapor sensor based on porous silicon was explored.

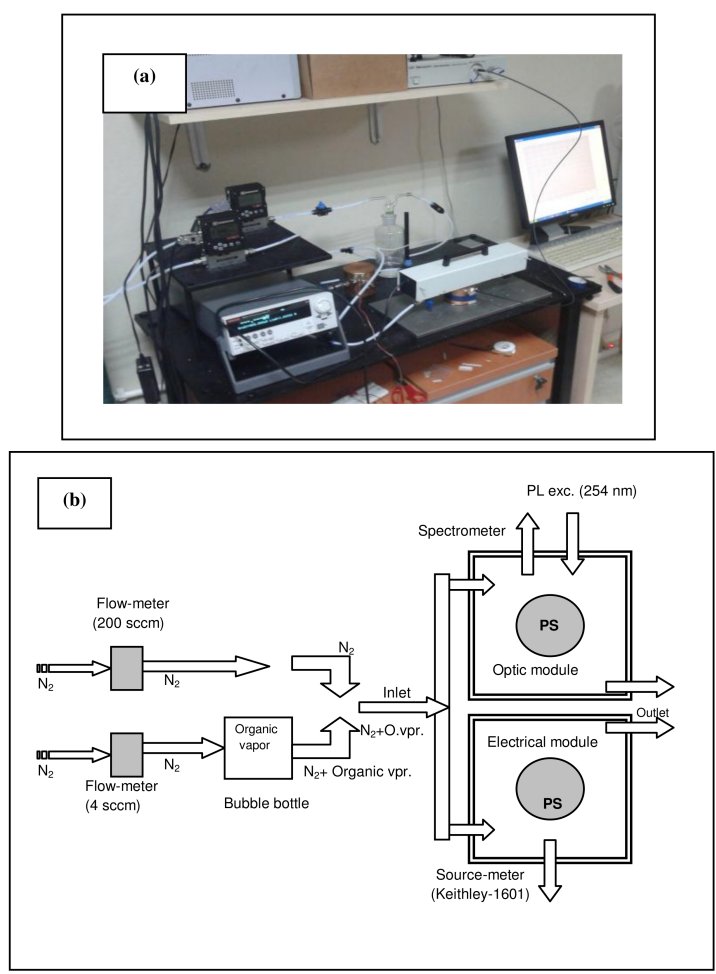

Fig. 1. Picture of the experimental set-up for sensing of organic vapors (a) and its schematic diagram (b). 


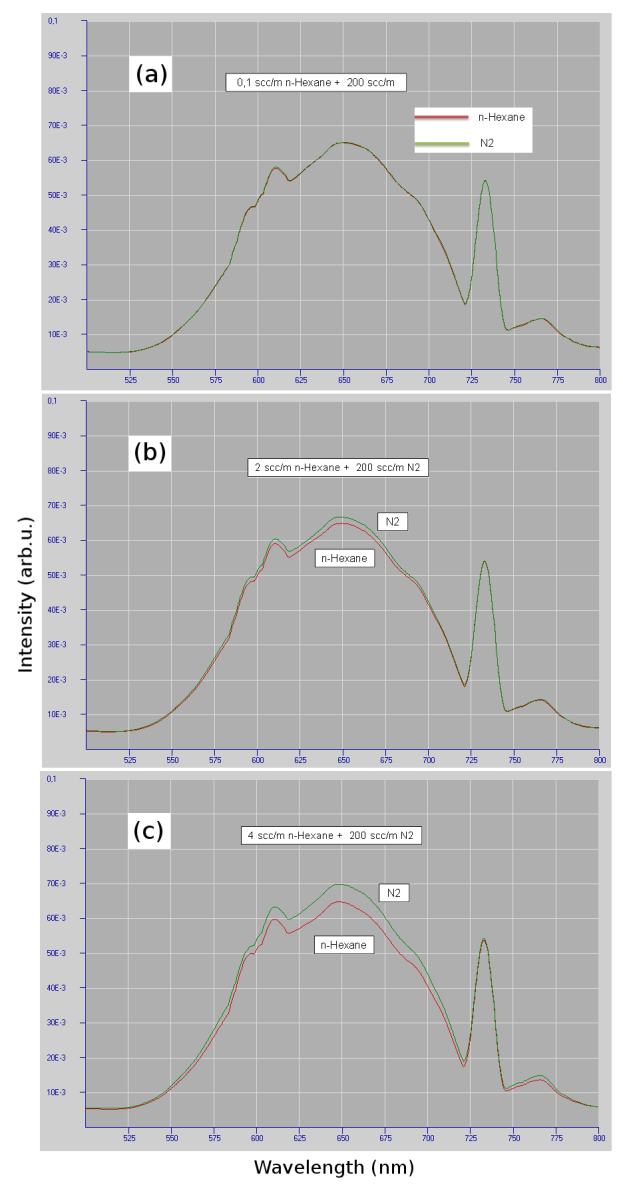

Fig. 2. The PL spectra of PS with respect to hexane concentration, (a) $0.1 \mathrm{sccm}$, (b) $2 \mathrm{sccm}$ and (c) $4 \mathrm{sccm}$. Green line is the PL spectrum during exposure to $\mathrm{N}_{2}$ alone, for five minutes, and red line is the PL spectrum during exposure to $\mathrm{N}_{2}+$ hexane mixture for five minutes.

\section{Experimental}

Details of electrochemical etching of crystalline silicon and PS production were given in details in Ref. 8. Schematic diagram and a picture of experimental setup for sensing vapors can be seen in Fig. 1. The PL quenching measurements of PS were performed in optical module and DC electrical measurements were performed in electrical module. Nitrogen gas $\left(\mathrm{N}_{2}\right)$ was used as a carrier gas for both modules. Two flow-meters from Sierra (smart track 100 and micro track 101), which have a maximum flow of $200 \mathrm{sccm}$ and $4 \mathrm{sccm}$ were used as nitrogen gas controllers. Nitrogen gas was separated into two lines. While one of the lines was connected to a bubble bottle to obtain organic vapor-gas $\left(\mathrm{N}_{2}\right)$ mix, the other line was connected to the pure $\mathrm{N}_{2}$ tube. $254 \mathrm{~nm}$ wave length of light from a UV lamp (Konrad Benda) was used for PL excitation. PS surface was coated with a metal inter-digital-electrode (IDE) (Aluminum, $0.2 \mathrm{~g}, 99.999 \%$ ) combined with thermal evaporation system for electrical measurements. Etching process and sensor measurements were carried out at room temperature.

\section{Results}

Nitrogen gas was introduced into the optic module first by a flow-meter $(200 \mathrm{sccm})$ and continuous PL spectra were then taken to obtain stable PL. After this, the other flow-meter $(4 \mathrm{sccm})$ which controls the organic vapor was run to obtain pre-determined $\mathrm{N}_{2}$-organic vapor mixture. The process was changed periodically $(5 \mathrm{~min})$ by altering the organic vapor concentrations. PS was waited for each period in optic module which was filled by pure $\mathrm{N}_{2}$ gas. Same process was repeated for the electrical module.

Figure 2 shows photoluminescence spectra of PS in response to various concentrations of hexane during exposure to vapor. It can be seen from the figure that PL intensity decreased during exposure, with the decreasing hexane vapor level. The other organic vapors displayed similar effect on PL spectra. It can clearly be seen that PL intensity depends on the concentration of hexane vapor, also showing that PS surface is rather sensitive to the vapors.

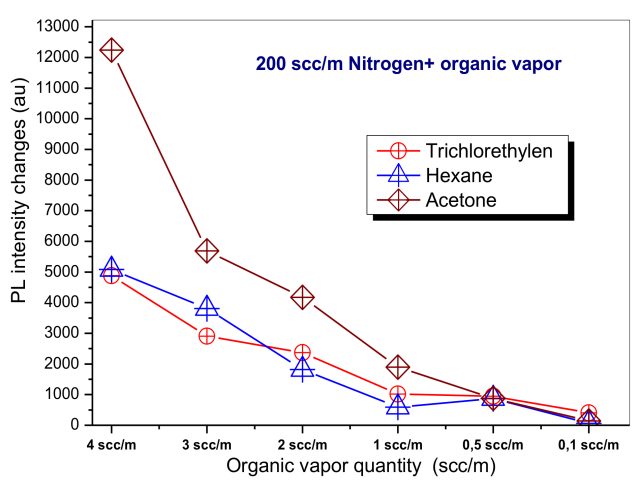

Fig. 3. Change in PL intensity with respect to organic vapor concentrations.

Figure 3 shows PL intensity changes with respect to organic vapor concentrations. It is seen from the figure that intensities of PL spectra decrease with decreasing organic vapor concentration, indicating that PS surface is more sensitive to acetone vapor than to hexane and trichloroethylene vapors.

Figure 4 shows electrical response of PS sensors during exposure to various concentrations of organic vapors. It is shown in the figure that the electrical signal increases during exposure to mixture of $\mathrm{N}_{2}+$ organic vapor and then decreases during exposure to carrier gas $\left(\mathrm{N}_{2}\right)$. This can be explained by capillary condensation of the vapors in pores of the PS, as was mentioned in [9]. Air in the pores of PS is replaced with the organic vapors when it is being exposed to vapors. Si-H bonds, which result from anodisation at the PS surface, also play a critical role in the organic vapor ad/absorption.

\section{Conclusions}

We propose a novel and simple way to use PS as an active material for sensing organic vapors such as trichloroethylene, hexane and acetone. The possibility 

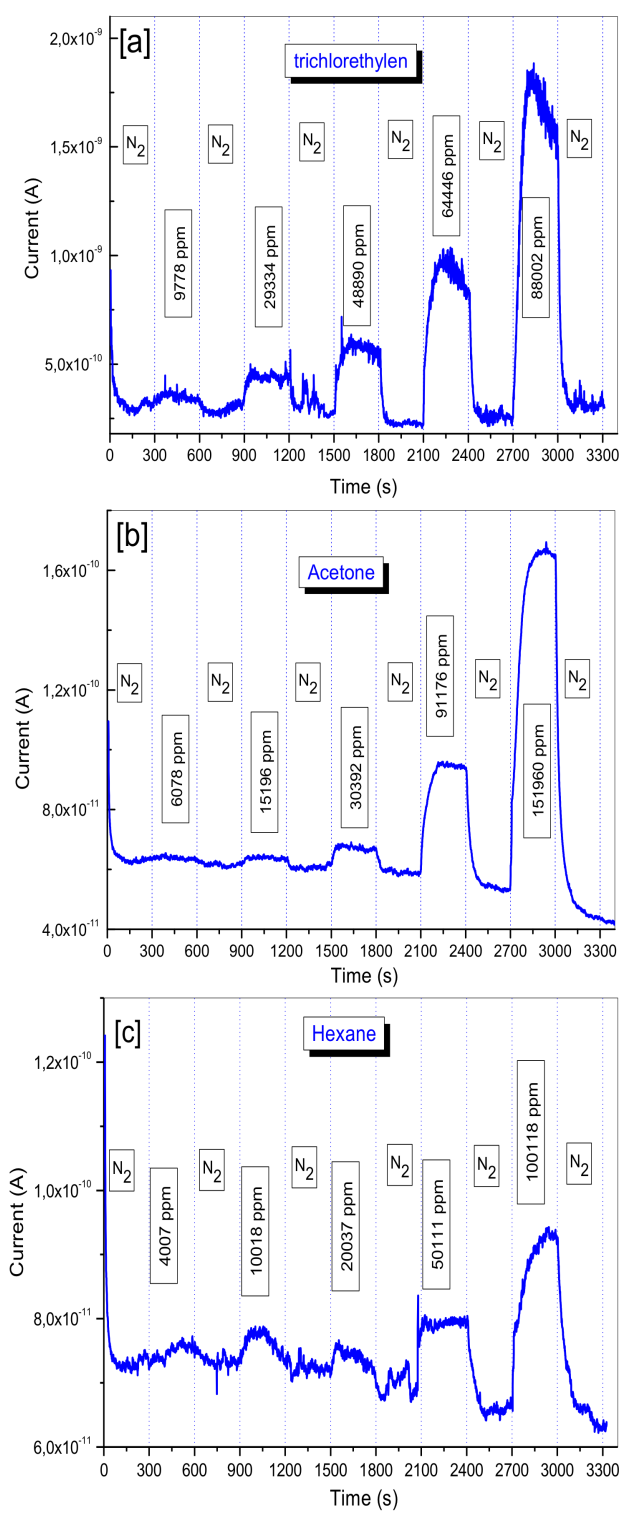

Fig. 4. The DC current response of the sensor to the organic vapors: trichloroethylene (a), acetone (b) and hexane (c).

of an organic vapor sensor based on porous silicon layer was demonstrated. Changes of PL intensity and resistivity with the organic vapor levels were investigated in this study. To the best of our knowledge, usage of the change of PL intensity as a sensing parameter has not been previously investigated with porous silicon.
We observed that PL intensity decreases, whereas, electrical signal (current) increases during exposure to increasing vapor concentrations, because of the capillary condensation of the organic vapors into the pores. The measurement results indicated that PS surface showed good vapor sensing performances with respect to organic vapors. It can therefore be concluded that $P S$ is an ideal candidate material for fabrication of high performance, selective electrical/optical vapor sensor in the near future. However, it is clear that a lot of research is needed before application of porous silicon as an organic vapor sensor.

\section{References}

[1] M. Mabrook, P. Hawkins, Sensors 2, 374 (2002).

[2] H.J Kim, Y.Y Kim, K.W Lee, Sensors and Actuators A: Phys. 165, 276 (2011).

[3] R. Çapan, Y. Açıkbaş, M. Evyapan, Mater. Lett. 61, 417 (2007).

[4] W.J. Salcedo, F.J.R Fernandez, J.C. Rubim, Spectrochimica Acta Part A 60, 1065 (2004).

[5] G. Barillaro, A. Nannini, F. Pieri, Sensors and Actutours B: Chem. 93, 263 (2003).

[6] T. Holec, T. Chvojka, I. Jelinek, J. Jindiric, I. Nemec, I. Pelant, J. Valenta, J. Dian, Mat. Sci. Eng. C 19, 251 (2002).

[7] H.K. Min, H.S. Yang, S.M. Cho, Sensor and Actuators B Chem. 67, 199 (2000).

[8] O. Yllmaz, E. Kayahan, F. Goren, F. Dumludag, Acta Physica Polonica A 125(2), 288 (2014).

[9] S. Dhanekar, S.S. Islam, T. Islam, A.K. Shukla, Harsh, Physica E 42, 1648 (2010). 